\title{
A model of degree of scattering polarization for oil spilling
}

\author{
XIAO WANG ${ }^{1}$, JIANG XU $^{1,2,}{ }^{*}$, WeIXIAN QIAN ${ }^{2}$, JiNGYI OUYANG $^{1}$ \\ ${ }^{1}$ The Higher Educational Key Laboratory of Optics-Electrics Application of Biomaterials, \\ School of Engineering, Jiangxi Agricultural University, \\ Nanchang, Jiangxi, 330045, China \\ ${ }^{2}$ National Defense Key Laboratory of Optoelectronic Engineering, \\ Nanjing University of Science and Technology, \\ Nanjing, Jiangsu, 210094, China \\ ${ }^{*}$ Corresponding author: xujiangstart@163.com
}

Oil slicks often show uncertain surface roughness and Fresnel reflection parameters. Consequently, differentiating oil spilled on the seawater in these areas using optical sensors is a challenge. Therefore, the optical mechanism of the oil film has been studied by the Maxwell equation. It is found that the polarization characteristics of the oil slicks can help us to overcome this problem. According to the Fresnel formula, the scattering coefficient and scattering rate of the homogeneous oil film have been deduced, and the phase difference of the scattering electromagnetic wave has also been calculated to verify the accuracy of the model. The parameter, a degree of scattering polarization, has been derived to identify the oil slicks on the sea wave. It depends on accurately knowing the Stokes parameter for the reflected light, and varies with the refractive index of the surface layer and viewing angles. The actual spilled oil has been measured by this model, and the oil film can be accurately identified at various angles. These preliminary results suggest that the potential of multi-angle polarization measurement of ocean surface needs further researches.

Keywords: optical mechanism, surface microstructure, degree of scattering polarization, Fresnel formulas, oil slicks.

\section{Introduction}

Marine oil spill accident could produce different impacts on marine ecological environment and social economy $[\underline{1}, 2]$. Oil pollution usually occurs around major shipping routes [ $\underline{3}$ ] and indicates close connection with offshore installations [4] , such as Deepwater Horizon oil spill accident in the Gulf of Mexico in 2010 [ㄷ]. As soon as the oil spill entered into seawater, the pollution of oil is very serious to the marine environment and marine biological resources. The oil spill on the sea will greatly reduce the exchange rate of oxygen between the seawater and atmosphere to destroy the ecological balance of the oceans $[\underline{6}, \underline{7}]$. 
Therefore, how to detect the oil spill on sea quickly and take corresponding measures is always the research focus of marine environment ecological security $[\underline{5}, \underline{8}-\underline{10}]$. At present, the oil spill detection methods are mainly of visible light, synthetic aperture radar, high spectrum, laser radar and so on [11- $\underline{13}]$. These methods are based on the information of scattering intensity from the material surface to identify the different kinds of objects in the measurement area. However, the oil spill spreads by gravity, wind and current to a bigger area and from the oil slick with different thickness on the ocean surface [14]. The structure of the surface becomes rough, and the measured value may exceed the threshold range of detectors [15-17]. The accuracy of traditional measurement techniques is affected by the observation angles.

For that reason, this paper has used the relationship between scattering angles and degree of polarization to derive the physical model of polarization scattering, and the degree of polarization of scattering has been extracted as the basis for detection of spilled oil on the sea. It is found that this theory compensates for the shortcomings of traditional measurement techniques, and it has been proved in the multi-angle measurements of spilled oil on the sea wave.

\section{Surface optical path of oil film}

Suppose that a plane wave is incident on a uniform oil film, the thickness of layered medium is $h$, and the two faces of the oil film are connected to a homogeneous half infinite medium, respectively. According to the Maxwell equation, we can get the following:

$$
\mathbf{E} \cdot \operatorname{curl} \mathbf{H}-\mathbf{H} \cdot \operatorname{curl} \mathbf{E}=\frac{4 \pi}{c} \dot{\mathbf{E}} \cdot \mathbf{E}+\frac{1}{c} \mathbf{j} \cdot \dot{\mathbf{D}}+\frac{1}{c} \mathbf{H} \cdot \dot{\mathbf{B}}
$$

where the two vectors, $\mathbf{E}$ and $\mathbf{B}$, are called the electric vector and the magnetic induction. And the second set of vectors are the electric current density $\mathbf{j}$, the electric displacement $\mathbf{D}$, and the magnetic vector $\mathbf{H}$. The dot is denoting differentiation with respect to time.

Also, by a well-known vector identity, the term on the left may be expressed as the divergence of the vector product of $\mathbf{H}$ and $\mathbf{E}$ :

$$
\mathbf{E} \cdot \operatorname{curl} \mathbf{H}-\mathbf{H} \cdot \operatorname{curl} \mathbf{E}=-\operatorname{div}(\mathbf{E} \times \mathbf{H})
$$

Then, multiply this equation by $c / 4 \pi$, integrate throughout an arbitrary volume and apply Gauss's theorem, this gives

$$
\frac{1}{4 \pi} \int(\mathbf{E} \cdot \dot{\mathbf{D}}+\mathbf{H} \cdot \dot{\mathbf{B}}) \mathrm{d} V+\int \mathbf{j} \cdot \mathbf{E} \mathrm{d} V+\frac{c}{4 \pi} \int(\mathbf{E} \times \mathbf{H}) \cdot \mathbf{n} \mathrm{d} S=0
$$

where the last integral is taken over the boundary of the volume, $\mathbf{n}$ being the unit outward normal. It represents the energy law of an electromagnetic field.

The boundary condition requires that the tangent component should be continuous when $\mathbf{E}$ passes the two boundaries of the layered medium. Therefore, we assume that 


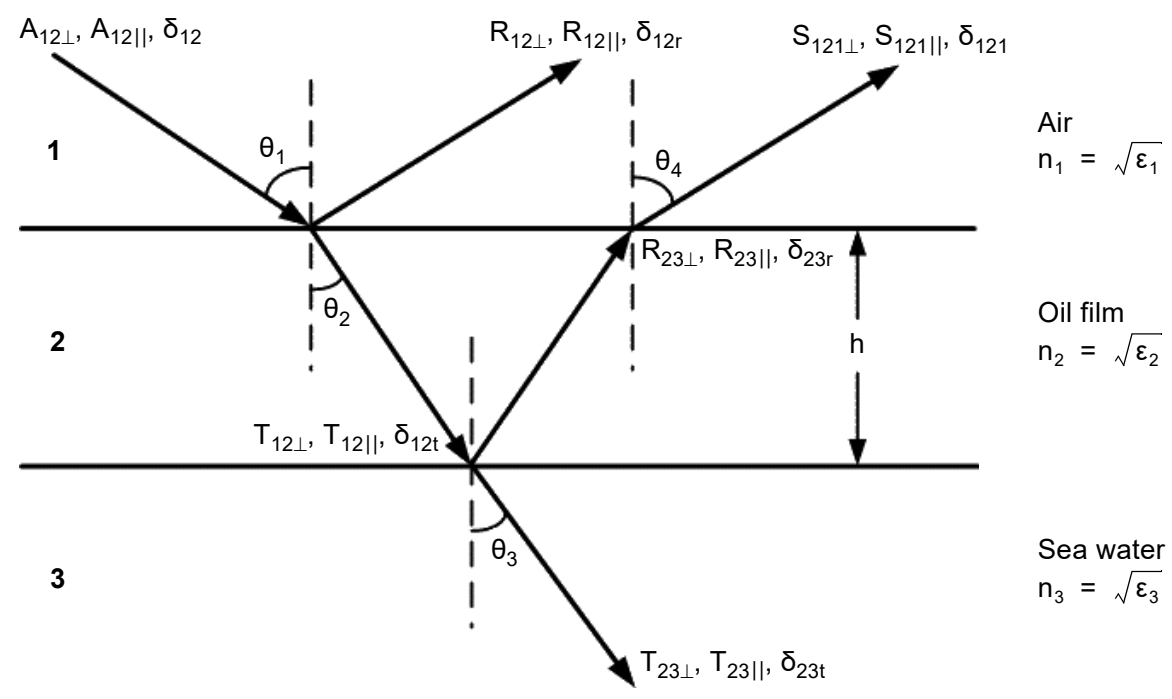

Fig. 1. The propagation of electromagnetic waves through a uniform oil film.

all the oil film media are nonmagnetic $(\mu=1)$. A schematic diagram of the single propagation of electromagnetic waves in a homogeneous oil film is shown in Fig. 1.

The empirical refractive index $n$ and extinction index $k$ of soil are 1.53 and 0.1280 , and the values of water are 1.33 and 0.0028 . Therefore, when the polarized light has irradiated to the surface of oil film by the incident angle of $\theta_{\mathrm{i}}\left(0^{\circ} \leq \theta_{\mathrm{i}}<90^{\circ}\right)$, the range of the refractive angle $\theta_{\mathrm{t}}$ is from $0^{\circ}$ to $40.81^{\circ}$, and $0 \leq \tan \theta_{\mathrm{t}}<0.8636$, then

$$
\lim _{h / d \rightarrow \infty}\left(\tan \theta_{\mathrm{t}}\right)^{2 h / d}=0
$$

Research shows that the first order reflected polarized light $\left(R_{12 \perp}, R_{12||}, \delta_{12}\right)$ and refractive polarized light $\left(T_{12 \perp}, T_{12||}, \delta_{12 t}\right)$ will emerge when the polarized light $\left(A_{12 \perp}\right.$, $\left.A_{12||}, \delta_{12}\right)$ illuminates the surface of oil spill. If the thickness of the oil film is greater than the diameter of molecular group, the collect signal of the second order refractive polarized light $\left(S_{121 \perp}, S_{121 \|}, \delta_{121}\right)$ is more weak, and the signal mainly comes from the first order reflected polarized light $\left(R_{12 \perp}, R_{12 \mid}, \delta_{12}\right)$. This is a strong basis for the optimization of a light path theory model.

\section{The polarization characteristics of oil film}

The characteristic matrix of a single homogeneous medium film can be expressed as $[\underline{18}, \underline{19}]$ :

$$
M(z)=\left[\begin{array}{lc}
\cos \left(k_{0} n z \cos \theta\right) & -\frac{i}{p} \sin \left(k_{0} n z \cos \theta\right) \\
-i p \sin \left(k_{0} n z \cos \theta\right) & \cos \left(k_{0} n z \cos \theta\right)
\end{array}\right]
$$


Denoting by subscripts 1, 2 and 3 quantities which refer to the three media (air, oil film and sea water). And

$$
\left\{\begin{array}{l}
m_{11}=m_{22}=\cos \beta \\
m_{12}=-\frac{i}{p_{2}} \sin \beta \\
m_{21}=-i p_{2} \sin \beta
\end{array}\right.
$$

where, $\beta=(2 \pi / \lambda) n_{2} h \cos \theta_{2}$ and $p_{j}=n_{j} \cos \theta_{j}(j=1,2,3)$.

According to the Fresnel formulae, the resulting formulae may be expressed in terms of the reflection coefficients $\left(r_{12}, r_{23}\right)$ and transmission coefficients $\left(t_{12}, t_{23}\right)$ at the first and the second surface, respectively. And

$$
\begin{aligned}
& \left\{\begin{array}{l}
r_{12}=\frac{n_{1} \cos \theta_{1}-n_{2} \cos \theta_{2}}{n_{1} \cos \theta_{1}+n_{2} \cos \theta_{2}} \\
t_{12}=\frac{2 n_{1} \cos \theta_{1}}{n_{1} \cos \theta_{1}+n_{2} \cos \theta_{2}}
\end{array}\right. \\
& \left\{\begin{array}{l}
r_{23}=\frac{n_{2} \cos \theta_{2}-n_{3} \cos \theta_{3}}{n_{2} \cos \theta_{2}+n_{3} \cos \theta_{3}} \\
t_{23}=\frac{2 n_{2} \cos \theta_{2}}{n_{2} \cos \theta_{2}+n_{3} \cos \theta_{3}}
\end{array}\right.
\end{aligned}
$$

Therefore, the expression of the scattering coefficient $s_{121}$ of the second scattering can be expressed as

$$
\begin{aligned}
s_{121} & =t_{12} r_{23} t_{21} \\
& =\frac{2 n_{1} \cos \theta_{1}}{n_{1} \cos \theta_{1}+n_{2} \cos \theta_{2}} \frac{n_{2} \cos \theta_{2}-n_{3} \cos \theta_{3}}{n_{2} \cos \theta_{2}+n_{3} \cos \theta_{3}} \frac{2 n_{2} \cos \theta_{2}}{n_{2} \cos \theta_{2}+n_{1} \cos \theta_{1}}
\end{aligned}
$$

And the scattering coefficient and scattering rate of the homogeneous oil film medium can be expressed as follows:

$$
\begin{aligned}
& s=\frac{r_{12}+s_{121}}{1+r_{12} s_{121}}=\frac{r_{12}+r_{23} \exp (2 i \beta)}{1+r_{12} r_{23} \exp (2 i \beta)} \\
& S=|s|^{2}=\frac{r_{12}^{2}+r_{23}^{2}+2 r_{12} r_{23} \cos (2 \beta)}{1+r_{12}^{2} r_{23}^{2}+2 r_{12} r_{23} \cos (2 \beta)}
\end{aligned}
$$


According to the Eqs. (10) and (11), the phase difference of the scattering electromagnetic wave can be calculated as:

$$
\delta_{\mathrm{s}}=\arg (s)=\frac{r_{23}\left(1-r_{12}^{2}\right) \sin (2 \beta)}{r_{12}\left(1+r_{23}^{2}\right)+r_{23}\left(1+r_{12}^{2}\right) \cos (2 \beta)}
$$

Therefore, under proper adjustment, the homogeneous oil film medium has the characteristics of filtering, which only lets through the spectrum in the specific range. And it can also change the phase of the incident light and have the polarization characteristic. These properties are related not only to the physical parameters of the medium itself, such as electrical conductivity, permeability, complex refractive index, but also to the angle of incident angle and the angle of observation. This conclusion has great significance for research and can be used for the detection of oil spill under the support of satellite remote sensing technology [20-22].

\section{The experimental device and method}

Model experiments were carried out in a dark room, and the measurement model of bidirectional reflectance distribution function was used to collect polarization data in this experiment. We used nine different incident angles $\left(35^{\circ}, 40^{\circ}, 45^{\circ}, 50^{\circ}, 55^{\circ}, 60^{\circ}\right.$, $65^{\circ}, 70^{\circ}$ and $75^{\circ}$ ) to measure the samples, and the measured angle was $90^{\circ}$. The light source was a helium-neon gas laser, and the wavelength was $632.8 \mathrm{~nm}$. The detector was a CCD camera (image size: $800 \times 600$ pixels for 4096 gray levels, and the physical size of detector is $5.27 \times 3.96 \mathrm{~mm}$ ). The CCD camera was used as a photometer [23]. It was a kind of CCD aperture photometry, and algorithms for CCD photometry were shown in Ref. [24]. The experimental apparatus was shown in Fig. 2.

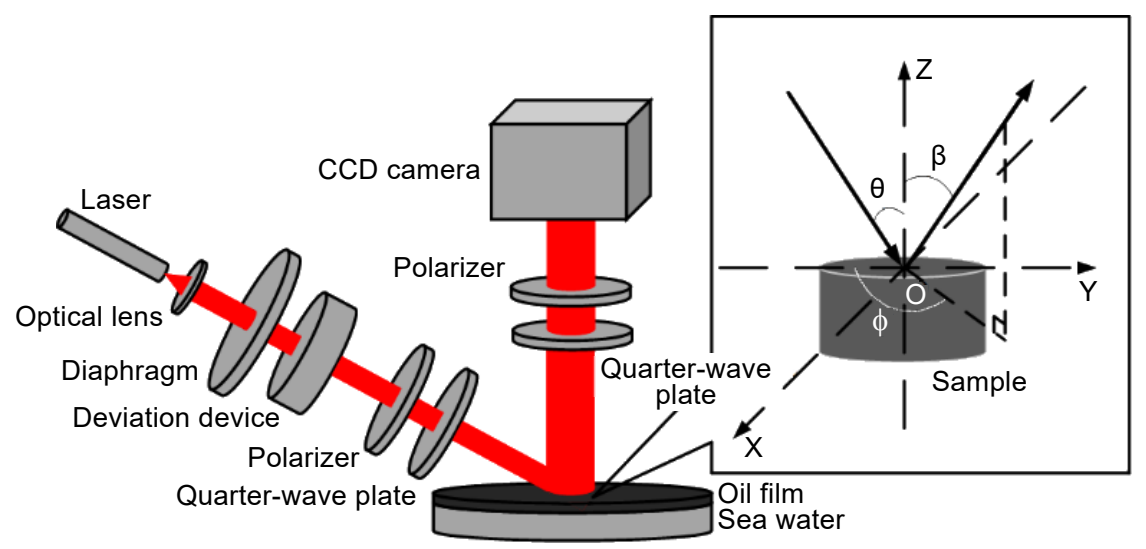

Fig. 2. The sketch map of polarization measurement. 
The experiment device is composed of a light source, optical lens, diaphragm, deviation device, polarizer, quarter-wave plate and CCD camera. The laser light is focused for a parallel beam by lens, and through a diaphragm with $1.88 \mathrm{~mm}$ circular aperture to be the controllable linear polarized light. Then the polarizer and quarter-wave plate are controlled to simulate different polarized lights with unique polarization states. According to the scattering principle, the signal of scattering light can be acquired by CCD camera which is through the quarter-wave plate and polarizer of receiving terminal. Finally, the Matlab software has been used to analyze the measurement data, the polarization parameters and Mueller matrixes of the samples. And the Ningxi oil, Yiyang oil and GII oil were used as the research samples with API values of 50, 31 and 22 , respectively.

\section{Experimental procedures}

\subsection{Decomposition of Mueller matrix}

Jones matrix or Mueller matrix can be used as the polarized state transformation matrix $[\underline{25}, \underline{26}]$. Optical equations are usually described by Jones matrix, such as Fresnel equations $[27,28]$. Therefore, Jones matrix and Mueller matrix are the good choices in material identification. However, Jones matrix can only detect the pure material and have no way to model rough surfaces samples whose polarized state transformations are mixed. Relatively, Mueller matrix can do it, but it is difficult to establish the physical model. In a real world application, the study on the mixed state is more valuable. From this idea, the Mueller matrix $M$ of this paper can then be decomposed as [29]

$$
M=\left[\begin{array}{llll}
S_{\|}^{2}+S_{\perp}^{2} & S_{\|}^{2}-S_{\perp}^{2} & 0 & 0 \\
S_{\|}^{2}-S_{\perp}^{2} & S_{\|}^{2}+S_{\perp}^{2} & 0 & 0 \\
0 & 0 & 2 S_{\|} S_{\perp} \cos \delta_{\mathrm{s}} & 2 S_{\|} S_{\perp} \sin \delta_{\mathrm{s}} \\
0 & 0 & -2 S_{\|} S_{\perp} \sin \delta_{\mathrm{s}} & 2 S_{\|} S_{\perp} \cos \delta_{\mathrm{s}}
\end{array}\right]+d M_{\mathrm{dp}}
$$

where $d$ is the coefficient of depolarization.

\subsection{Polarization parameters}

The simulation equation is derived by Fresnel equations:

$$
P \exp \left(i \delta_{\mathrm{s}}\right)=\frac{2 \sin \theta \tan \theta}{\sin \theta \tan \theta-\sqrt{n^{2}-k^{2}-\sin ^{2} \theta+2 n k i}}-1
$$

where $n$ is the complex refractive index, $k$ is extinction index, and $\theta$ is incident angle. While $P$ can be calculated from absolute value of $P \exp \left(i \delta_{\mathrm{s}}\right)$, and $\delta_{\mathrm{s}}$ can be calculated from phase angles of $P \exp \left(i \delta_{\mathrm{s}}\right)$. 
$\mathrm{T}$ a b 1 e. The refractive index $n_{\mathrm{i}}$ and refraction angle $\theta_{\mathrm{t}}$ of water and oil film's surface.

\begin{tabular}{llllllll}
\hline $\begin{array}{l}\text { Incident } \\
\text { angles } \theta_{\mathrm{i}}\end{array}$ & $\begin{array}{l}\text { Refractive } \\
\text { index of } \\
\text { water } n_{3}\end{array}$ & $\begin{array}{l}\text { Refractive } \\
\text { index of } \\
\text { Ningxi oil }\end{array}$ & $\begin{array}{l}\text { Refraction } \\
\text { angle of } \\
\text { Ningxi oil }\end{array}$ & $\begin{array}{l}\text { Refractive } \\
\text { index of } \\
\text { Yiyang oil }\end{array}$ & $\begin{array}{l}\text { Refraction } \\
\text { angle of } \\
\text { Yiyang oil }\end{array}$ & $\begin{array}{l}\text { Refractive } \\
\text { index of } \\
\text { GII oil }\end{array}$ & $\begin{array}{l}\text { Refraction } \\
\text { angle of } \\
\text { GII oil }\end{array}$ \\
\hline $35^{\circ}$ & 1.3130 & 1.5070 & $23.9132^{\circ}$ & 1.4715 & $24.5276^{\circ}$ & 1.5046 & $23.9537^{\circ}$ \\
$40^{\circ}$ & 1.3257 & 1.5258 & $27.2292^{\circ}$ & 1.4599 & $28.5682^{\circ}$ & 1.5041 & $27.6554^{\circ}$ \\
$45^{\circ}$ & 1.3156 & 1.4958 & $31.6729^{\circ}$ & 1.4731 & $32.2192^{\circ}$ & 1.5002 & $31.5693^{\circ}$ \\
$50^{\circ}$ & 1.3449 & 1.4908 & $35.8289^{\circ}$ & 1.5008 & $35.5537^{\circ}$ & 1.4999 & $35.5783^{\circ}$ \\
$55^{\circ}$ & 1.3398 & 1.5031 & $39.6901^{\circ}$ & 1.5213 & $39.1235^{\circ}$ & 1.5080 & $39.5357^{\circ}$ \\
$60^{\circ}$ & 1.3562 & 1.5302 & $43.1848^{\circ}$ & 1.5375 & $42.9300^{\circ}$ & 1.5229 & $43.4431^{\circ}$ \\
$65^{\circ}$ & 1.3237 & 1.5741 & $46.1128^{\circ}$ & 1.6061 & $44.9385^{\circ}$ & 1.5420 & $47.3672^{\circ}$ \\
$70^{\circ}$ & 1.3366 & 1.5874 & $50.3218^{\circ}$ & 1.6141 & $49.1927^{\circ}$ & 1.5607 & $51.5185^{\circ}$ \\
$75^{\circ}$ & 1.3351 & 1.5949 & $55.1589^{\circ}$ & 1.5750 & $56.2128^{\circ}$ & 1.6148 & $54.1570^{\circ}$ \\
\hline
\end{tabular}

With the aid of a photometer (THORLABS-PM100D), the vertical and horizontal light intensity value of incident polarized light were measured: $A_{12 \perp}=0.348 \mathrm{~mW}$, $A_{12||}=0.399 \mathrm{~mW}$. Then the polarizer and quarter-wave plate were used to simulate different polarized lights with unique polarization parameters. The refractive indexes of water and oil film were calculated in different measuring angles by the Fresnel formula, and the results are shown in the Table.

According to the Eq. (14), we could calculate the amplitude ratio $P$ and phase retardation $\delta_{\mathrm{s}}$, and the results can be seen in Fig. 3. In order to make the parameters of polarized light signal more precise, this experiment had collected 36 groups of measurement data. And the 16 unknown variables (Mueller matrix) would be solved by using 36 equations.

The amplitude ratio and phase retardation are almost unchanged. The pink inverted triangles are the polarization parameters of the seawater, and the others are the measured value of the three kinds of oil slicks. With the increase of measured angles, the amplitude

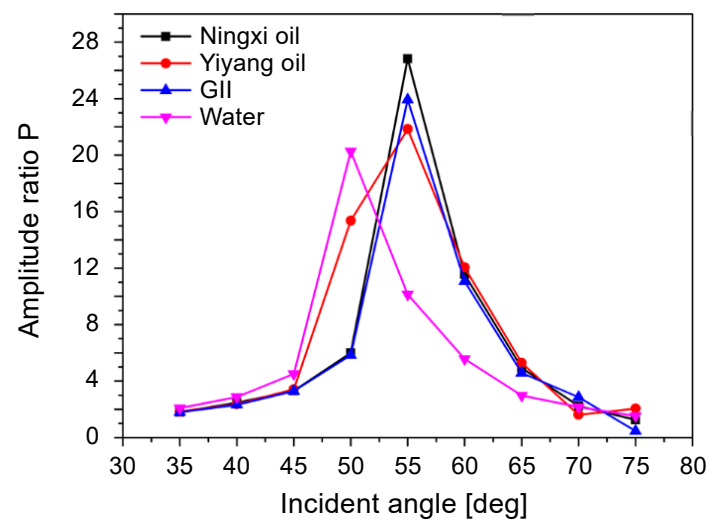

a

Fig. 3. The amplitude ratio (a) and phase retardation (b) of incident polarized light and scattered polarized light with different incident angles. 


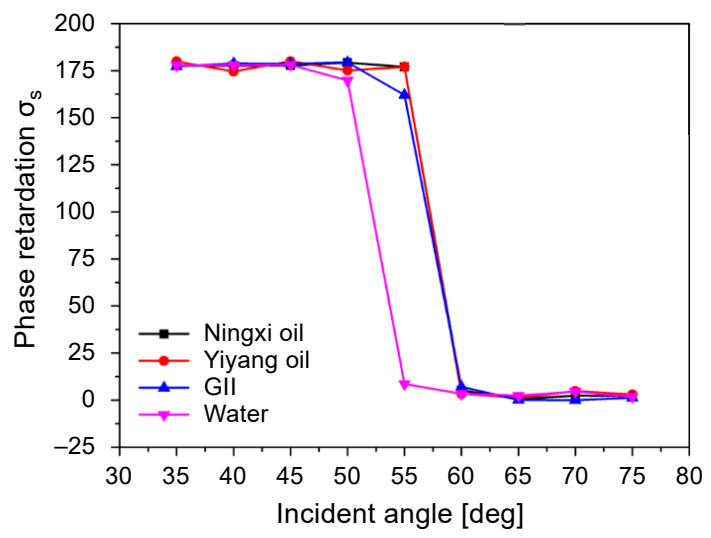

b

Fig. 3. Continued.

ratio and phase retardation change at Brewster angles $\left(\theta_{\mathrm{B} \text {, water }}=53.06^{\circ}, \theta_{\mathrm{B} \text {, oil }}=56.83^{\circ}\right)$. In the small measured angles, the amplitude ratios of the four kinds of polarized light are similar, the phase retardations of scattering polarized light of water and oil film differ by $\pi$. But the two polarized parameters have a sudden change at Brewster angles, the amplitude ration reaches a maximum, and the phase retardation drops to $0^{\circ}$. These results show that the parameters of incident polarized light have been changed by the samples, and this feature could be used as the basis to identify different objects.

\section{The degree of polarization of scattering wave}

The Stokes vector is a $4 \times 1$ matrix which can be used to describe the polarization effect of electromagnetic wave and the samples [30]. Therefore, in this study we used the polarization information of the Stokes vector to analyze the change rules of polarization state of the incident polarized light.

Since the four Stokes parameters describe the radiance of light, the ratio of the intensity of the polarized portion to the total intensity is called the degree of polarization (DOP) of the wave, and

$$
\mathrm{DOP}=\frac{I_{\mathrm{pol}}}{I_{\mathrm{tot}}}=\frac{\sqrt{Q^{2}+U^{2}+V^{2}}}{I}=\frac{\left|S_{\|}^{2}-S_{\perp}^{2}\right|}{\left|S_{\|}^{2}+S_{\perp}^{2}\right|}
$$

where $I, Q, U$ and $V$ are the four parameters of Stokes vector; $S_{\|}, S_{\perp}$ are the horizontal and vertical components of the Stokes vector, respectively. According to the Eqs. (9)-(11), we find that the DOP is also related to the incident angle of the plane wave. Therefore, in the process of measuring the oil spill on the real sea, we must consider the effect of the rough sea surface on the measurement of DOP.

The surface of a rough oil slick (or of rough oil-free seawater) can be considered to a combination of tilted wave facets that also satisfy the law of Snell, which can be seen in Fig. 4. Real-world rough oil slicks and oil-free seawater surfaces can be ap- 


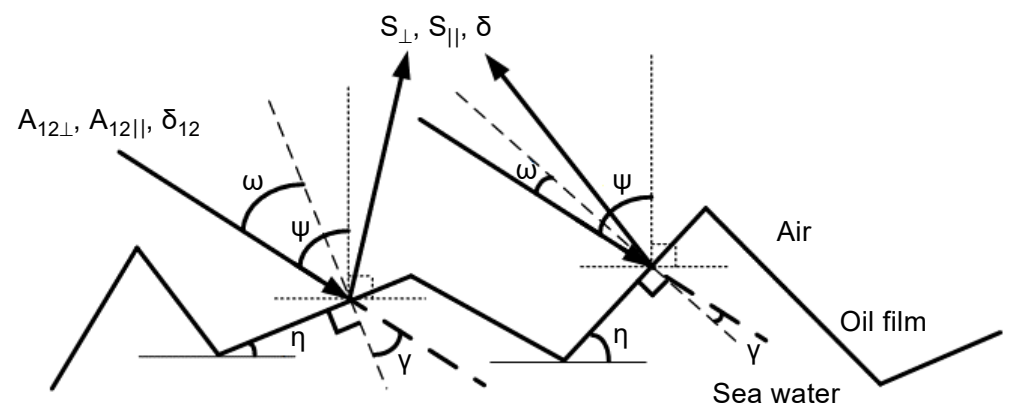

Fig. 4. Schematic of the Fresnel reflection of incident light on rough oil slicks and seawater surfaces (for polarizations orthogonal and parallel to the incident plane).

proximated as containing a number of tilted wave facets that satisfy both the laws of Fresnel and Snell. The Cox-Munk model quantitatively describes the size, shape, and intensity of the sun-glint pattern of these facets' combination through a probability distribution function (PDF) [31- $\underline{33}$, which can be expressed as follows:

$$
P\left(\psi, \varphi, \phi, \sigma^{2}\right)=\frac{1}{\pi \sigma^{2}} \exp \left(\frac{-\tan ^{2} \eta}{\sigma^{2}}\right)
$$

Where $\tan ^{2} \eta$ is given by

$$
\tan ^{2} \eta=\frac{\sin ^{2} \psi+\sin ^{2} \varphi+2 \sin \psi \sin \varphi \cos \phi}{(\cos \psi+\cos \varphi)^{2}}
$$

and, $\psi$ is the solar zenith angle, $\varphi$ is the sensor zenith angle, $\phi$ is the relative azimuth angle, $\sigma^{2}$ is the surface roughness variance (the sea surface roughness variance can be written as a function of the surface wind speed $\left.(w, \mathrm{~m} / \mathrm{s}), \sigma_{\text {water }}^{2}=0.003+0.00512 w[\underline{31}]\right)$, and $\eta$ is the tilt angle relative to the horizontal plane of a facet on the surface.

For a tilted wave facet, the local angle of the incident sunlight $\omega$ or the local reflection angle $(\omega)$ of the sun-glint specular reflection can be obtained from the spherical law of cosines: $\cos 2 \omega=\cos \psi \cos \varphi+\sin \psi \sin \varphi \cos \phi$. The local refraction angle $\gamma$ of the tilted wave facets (for an oil slick or oil-free seawater) can then be derived from the refractive indices of the oil slick or oil-free seawater. The radiance that results from a reflection of the sea surface varies with the surface roughness. The surface roughness Stokes parameter must be taken into consideration when simulating this real sea surface.

The Stokes parameters of the sun-glint reflected from the oil slick or the oil-free seawater surface are given by the following equation:

$$
\left[\begin{array}{llll}
I_{\mathrm{o}} & Q_{\mathrm{o}} & U_{\mathrm{o}} & V_{\mathrm{o}}
\end{array}\right]^{\mathrm{T}}=\frac{P\left(\psi, \varphi, \phi, \sigma^{2}\right)\left(1+\tan ^{2} \eta\right)}{4 \cos \varphi} R\left(\psi_{1}\right) M R\left(\psi_{2}\right)\left[\begin{array}{llll}
I_{\mathrm{i}} & Q_{\mathrm{i}} & U_{\mathrm{i}} & V_{\mathrm{i}}
\end{array}\right]^{\mathrm{T}}
$$

where $R$ is the rotation matrix, $\psi_{1}$ and $\psi_{2}$ are the two rotation angles. 


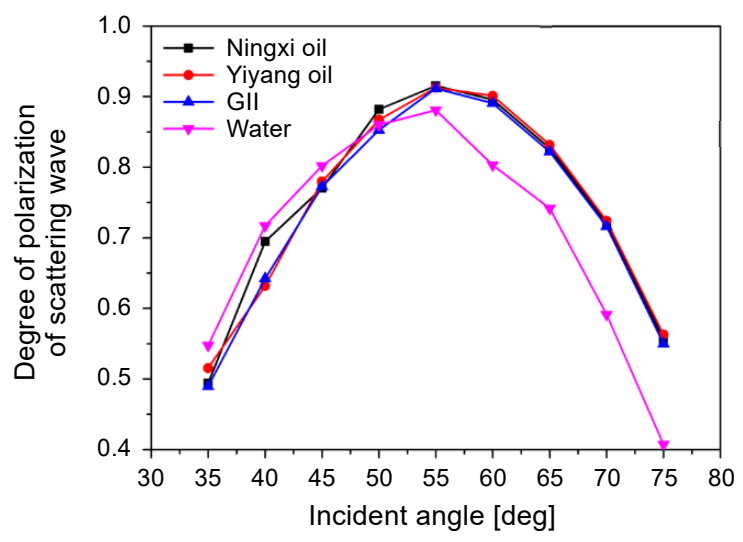

Fig. 5. Degree of polarization (DOP) of scattered polarized light with different incident angles.

We performed experiments using oil spilled on the sea to analyze DOP. The conclusion was shown in Fig. 5.

By analyzing the measured values of different incidence angles, we could find the following rules: 1) the DOPs of the three kinds of oil film are almost identical, and there is a significant difference from that of sea water; 2) the Brewster angle as the dividing line, when the incident angle is small, the optical propagation in the oil film becomes deeper, the weak scattering energy is collected by the detector, and the DOPs are lower than that of water; 3 ) when the incident angle is large, the optical path in the oil film becomes longer, and the multiple scattering between oil particles becomes very active, the DOPs are greater than that of sea water.

These features provide a very favorable basis for our identification of the oil spilled on the sea. The diameters of water and oil's molecules are only dozens of nanometers, and less than one-tenth of the wavelength of incident light, thus it is easy to produce the Rayleigh scattering. However, the wavelength of experimental incident light was $632.8 \mathrm{~nm}$, so the Rayleigh scattering would not be obvious in our experiment [34]. At the micro-level, we have found that the inter-atomic forces of the oil film were stronger than that of water, which made the surface of oil film more akin to a mirror, and the scattering effect was poorer. Therefore, in the range of large angles, the DOP of oil film would be greater than that of water.

\section{Conclusion}

We have established an analytical model of polarizing optical detection technology to identify the oil spill on the sea, and the polarization characteristics of measured samples have been proven by different polarized states of incident light and scattering light. Firstly, the optical path in the oil film has been analyzed, and the optical information of the single scattering and multiple scattering has been obtained. The polarization characteristics of the oil film has been verified by the Maxwell equation. Secondly, the characteristic matrix of a single homogeneous dielectric film has been studied. Ac- 
cording to the Fresnel formula, the scattering coefficient and scattering rate of the homogeneous oil film have been deduced, and the phase difference of the scattering electromagnetic wave has also been calculated. Then, we measured four kinds of samples and observed the polarization information of scattered light from nine different angles. The validity of the experimental data have been verified by the Fresnel formula. Finally, the degree of polarization of scattering has been studied, the relationship between the roughness of oil slicks and the degree of polarization of scattering have been analyzed, and these rules have been explained in detail by the experimental data. The results demonstrate that the proposed method is correct and more accurate and efficient performance can be achieved, thus it is more suitable for the identification and evaluation of oil spill on the sea.

Acknowledgments - This work was supported by the National Natural Science Foundation of China, Nos. 61675099, 61701233; the Fundamental Research Funds for the Central Universities, No. 30917011321.

\section{References}

[1] Brekke C., Solberg A.H.S., Oil spill detection by satellite remote sensing, Remote Sensing of Environment 95(1), 2005, pp. 1-13, DOI: 10.1016/j.rse.2004.11.015.

[2] Caballero A., Espino M., Sagarminaga Y., Ferrer L., Uriarte A., Gonzalez M., Simulating the migration of drifters deployed in the Bay of Biscay, during the Prestige crisis, Marine Pollution Bulletin 56(3), 2008, pp. 475-482, DOI: 10.1016/j.marpolbul.2007.11.005.

[3] Lu J., Marine oil spill detection, statistics and mapping with ERS SAR imagery in south-east Asia, International Journal of Remote Sensing 24(15), 2003, pp. 3013-3032, DOI: $\underline{10.1080 / 01431160110}$ $\underline{076216 .}$.

[4] Espedal H.A., Johannessen O.M., Detection of oil spills near offshore installations using synthetic aperture radar (SAR), International Journal of Remote Sensing 21(11), 2000, pp. 2141-2144, DOI: $10.1080 / 01431160050029468$.

[5] Mariano A.J., Kourafalou V.H., Srinivasan A., Kang H., Halliwell G.R., Ryan E.H., Roffer M., On the modeling of the 2010 Gulf of Mexico Oil Spill, Dynamics of Atmospheres and Oceans 52(1-2), 2011, pp. 322-340, DOI: 10.1016/j.dynatmoce.2011.06.001.

[6] Corsellis Y.Y., Krasovec M.M., Sylvi L.L., Cuny P.P., Militon C.C., Oil removal and effects of spilled oil on active microbial communities in close to salt-saturation brines, Extremophiles 20(3), 2016, pp. 235-250, DOI: 10.1007/s00792-016-0818-X.

[7] Miralles G., Nerini D., Mante C., Acquaviva M., Doumene P., Michotey V., Nazaret S., BERTRAND J., CUNY P., Effects of spilled oil on bacterial communities of Mediterranean coastal anoxic sediments chronically subjected to oil hydrocarbon contamination, Microbial Ecology 54(4), 2007, pp. 646-661, DOI: 10.1007/s00248-007-9221-6.

[8] Jha M.N., Levy J., GaO Y., Advances in remote sensing for oil spill disaster management: state-of -the-art sensors technology for oil spill surveillance, Sensors 8(1), 2008, pp. 236-255, DOI: 10.3390 / $\underline{\mathrm{s} 8010236}$.

[9] Kessler J.D., Valentine D.L., Redmond M.C., Du M., Chan E.W., Mendes S.D., Quiroz E.W., Villanueva C.J., Shusta S.S., Werra L.M., Yvon-Lewis S.A., Weber T.C., A persistent oxygen anomaly reveals the fate of spilled methane in the Deep Gulf of Mexico, Science 331(6015), 2011, pp. 312-315, DOI: 10.1126/science.1199697.

[10] LeIFER I., CLARK J.F., Chen R.F., Modifications of the local environment by natural marine hydrocarbon seeps, Geophysical Research Letters 27(22), 2000, pp. 3711-3714, DOI: 10.1029/2000GL011619. 
[11] Lu Y., Li X., Tian Q., Han W., An optical remote sensing model for estimating oil slick thickness based on two-beam interference theory, Optics Express 20(22), 2012, pp. 24496-24504, DOI: 10.1364/ OE.20.024496.

[12] Lu Y., Zhan W., Hu C., Detecting and quantifying oil slick thickness by thermal remote sensing: a ground-based experiment, Remote Sensing of Environment 181, 2016, pp. 207-217, DOI: 10.1016/ j.rse.2016.04.007.

[13] Garcia-Garrido V.J., Ramos A., Mancho A.M., Coca J., Wiggins S., A dynamical systems perspective for a real-time response to a marine oil spill, Marine Pollution Bulletin 112(1-2), 2016, pp. 201-210, DOI: 10.1016/j.marpolbul.2016.08.018.

[14] ZHong Z.X., You F.Q., Oil spill response planning with consideration of physicochemical evolution of the oil slick: a multiobjective optimization approach, Computers \& Chemical Engineering 35(8), 2011, pp. 1614-1630, DOI: 10.1016/j.compchemeng.2011.01.009.

[15] Tuchin V.V, Polarized light interaction with tissues, Journal of Biomedical Optics 21(7), 2016, article 071114, DOI: 10.1117/1.JBO.21.7.071114.

[16] Tuchin V.V., Wang L., Zimnyakov D.A., Optical Polarization in Biomedical Applications, Springer, Berlin, Heidelberg, 2006, DOI: 10.1007/978-3-540-45321-5.

[17] Wan M., Gu G., Qian W., Ren K., Chen Q., Stokes-vector-based polarimetric imaging system for adaptive target/background contrast enhancement, Applied Optics 55(21), 2016, pp. 5513-5519, DOI: $10.1364 / \mathrm{AO} .55 .005513$.

[18] Li H., Li L., ZHANG J., Multi-focus image fusion based on sparse feature matrix decomposition and morphological filtering, Optics Communications 342, 2015, pp. 1-11, DOI: 10.1016/j.optcom.2014.12.048.

[19] DufFin R.J., On the characteristic matrices of covariant systems, Physical Review 54(12), 1938, p. 1114, DOI: 10.1103/PhysRev.54.1114.

[20] Xu J., Qian W.X., Chen Q., Zhou Y., Calculation model of Hyperion reflected light scattering efficiency, Applied Optics 56(18), 2017, pp. 5248-5252, DOI: 10.1364/AO.56.005248.

[21] Xu J., Qian W.X., Lu D.M., Lu Y.C., Calculating model for equivalent consumption efficiency in polarization measurement system of oil-spilled on the sea, Infrared Physics \& Technology 77, 2016, pp. 474-479, DOI: 10.1016/j.infrared.2016.07.005.

[22] Xu J., Qian W.X., Chen Q., Gu G.H., Lu Y.C., Zhou Y., Uniform scattering power for monitoring the spilled oil on the sea, IEEE Photonics Journal 10(1), 2018, pp. 6100310, DOI: 10.1109/JPHOT. 2018.2799206.

[23] Le Roy-Brehonnet F., Le Jeune B., Utilization of Mueller matrix formalism to obtain optical targets depolarization and polarization properties, Progress in Quantum Electronics 21(2), 1997, pp. 109 -151, DOI: 10.1016/S0079-6727(97)84687-3.

[24] Mighell K.J., Algorithms for CCD Stellar Photometry, Astronomical Data Analysis Software and Systems VIII, ASP Conference Series, Vol. 172, [Eds.] D.M. Mehringer, R.L. Plante, D.A. Roberts, 1999, pp. 317-328.

[25] Ortega-Quijano N., Arce-Diego J.L., Mueller matrix differential decomposition, Optics Letters 36(10), 2011, pp. 1942-1944, DOI: 10.1364/OL.36.001942.

[26] Devlaminck V., Terrier P., Charbois J.M., Physically admissible parameterization for differential Mueller matrix of uniform media, Optics Letters 38(9), 2013, pp. 1410-1412, DOI: 10.1364/OL.38. $\underline{001410 .}$

[27] Born M., Principles of Optics, Publishing House of Electronics Industry, Beijing, 1999.

[28] Sharma K.K., Optics Principles and Applications, Academic Press, New York, Boston, 2006.

[29] Qian W., Zhou X., Lu Y., Xu J., Paths correlation matrix, Optics Letters 40(18), 2015, pp. 4336-4339, DOI: $10.1364 /$ OL.40.004336.

[30] Sowmya K., Nagendra K.N., Sampoorna M., Stenflo J.O., Polarized scattering of light for arbitrary magnetic fields with level-crossings from the combination of hyperfine and fine structure splittings, The Astrophysical Journal 814(2), 2015, p. 127, DOI: 10.1088/0004-637X/814/2/127. 
[31] Cox C., Munk W., Measurement of the roughness of the sea surface from photographs of the sun's glitter, Journal of the Optical Society of America 44(11), 1954, pp. 838-850, DOI: $\underline{10.1364 / \text { JOSA. }}$ 44.000838 .

[32] JACKSON C.R., ALPERS W., The role of the critical angle in brightness reversals on sunglint images of the sea surface, Journal of Geophysical Research: Oceans 115(C9), 2010, article C09019, DOI: $10.1029 / 2009 \mathrm{JC} 006037$.

[33] ZhANG H., WANG M.H., Evaluation of sun glint models using MODIS measurements, Journal of Quantitative Spectroscopy and Radiative Transfer 111(3), 2010, pp. 492-506, DOI: $\underline{10.1016 / j . j q s r t .}$ 2009.10.001.

[34] Young A.T., Rayleigh scattering, Applied Optics 20(4), 1981, pp. 533-535, DOI: 10.1364/AO.20. $\underline{000533}$. 